\title{
Reprodutibilidade do testes de sobrecarga hídrica
}

\author{
Reproducibility of water drinking test
}

\section{Caro Editor,}

Gostaríamos de congratular os autores pelo interesse em estudar o teste de sobrecarga hídrica (TSH) no glaucoma. (Medina FM, Rodrigues FK, Filho Pde T, Matsuo T, Vasconcellos JP, Costa VP. Reproducibility of water drinking test performed at different times of the day. Arq Bras Oftalmol. 2009;72(3):283-90)

Nos últimos anos nosso grupo avaliou o TSH em diversas situações relacionadas ao controle pressórico em pacientes com diagnóstico já estabelecido e submetidos ou não a terapia clínica e (ou) cirúrgica, com diferentes níveis de dano glaucomatoso e com evoluções diferentes em relação à progressão da doença ${ }^{(1-11)}$. A utilização do TSH para avaliar controle pressórico em olhos sob terapia clínica também passou a ser estudada por centros de pesquisa em outras partes do mundo como Estados Unidos e Europa ${ }^{(12-18)}$.

Particularmente em relação ao estudo apresentado pelos autores para avaliar a reprodutibilidade do TSH, gostaríamos de fazer algumas considerações.

Os autores apresentam uma certa preocupação em medir a reprodutibilidade do teste em horários diferentes para definir sua aplicabilidade nessas circunstâncias.

Em seu estudo, Medina et al. sugerem que a reprodutibilidade do TSH realizado em diferentes horários do dia é pobre e representa uma limitação à sua aplicabilidade para o diagnóstico e seguimento de pacientes com glaucoma.

No entanto, ao analisar os resultados dos autores, não pudemos deixar de notar que $80 \%$ dos pacientes com diagnóstico de glaucoma reproduziram picos pressóricos em dias e horários diferentes com variação menor do que $3 \mathrm{mmHg}$ (valor a partir do qual os autores consideram a diferença clinicamente relevante). Isso significa em outras palavras que 12 de 15 pacientes reproduziram o pico pressórico e que em apenas em 3 pacientes, o pico mostrou-se com reprodutibilidade clinicamente inaceitável. Ao nosso ver esses resultados, ao contrário do que os autores concluiram, mostram-se expressivos do ponto de vista clínico.

Também é importante que se saliente que este teste há muito tempo não é utilizado para o diagnóstico do glaucoma, devido ao elevado número de falsos positivos e falsos negativos e não pela possível falta de reprodutilidade do método quando feito em horários e dias diferentes como sugerem os autores. Nosso grupo tem utilizado o TSH para estudar o perfil pressórico dos pacientes, servindo como uma forma indireta de avaliar a facilidade de escoamento.

Concordamos com os autores que atualmente a curva tensional diária $24 \mathrm{~h}$, apesar de pouco utilizada pela sua dificulda- de prática, continua sendo o método padrão-ouro para acessar o perfil pressórico ao longo do dia em pacientes com glaucoma. Até o momento não existem estudos que tenham avaliado sua reprodutibilidade pelo teste de Bland-Altmann e nem por isto este método perdeu sua importância no arsenal de avaliação clínico.

Nosso grupo teve a oportunidade de avaliar a reprodutibilidade da CTD $24 \mathrm{~h}$ por outros métodos no passado ${ }^{(19-20)}$. Em 2 estudos foi observado que aproximadamente $75 \%$ dos picos da CTD 24 h realizada em dias consecutivos, apresentaram variação maior do que $20 \%$ semelhante aos achados dos autores para o TSH.

Em resumo, o número expressivo de trabalhos e editoriais publicados recentemente em vários centros internacionais e com centenas de pacientes estudados sugerem a potencialidade deste teste em fornecer informações complementares importantes na avaliação do controle pressórico de pacientes glauco$\operatorname{matosos}^{(1-18,21-22)}$.

Roberto M. Vessani
Marcelo Hatanaka
Ricardo Suzuki
Paulo Gelmann
Roberto F. S. Malta
Felipe A. Medeiros
Remo Susanna Jr.

\section{REFERÊNCIAS}

1. Medeiros FA, Pinheiro A, Moura FC, Leal BC, Susanna R Jr. Intraocular pressure fluctuations in medical versus surgically treated glaucomatous patients. J Ocul Pharmacol Ther. 2002;18(6):489-98.

2. Susanna R Jr, Medeiros FA, Vessani RM, Giampani J Jr, Borges AS, Jordão ML. Intraocular pressure fluctuations in response to the water-drinking provocative test in patients using latanoprost versus unoprostone. J Ocul Pharmacol Ther. 2004;20(5):401-10.

3. Susanna R Jr, Sheu WP; Latin American Glaucoma Society. Comparison of latanoprost with fixed-combination dorzolamide and timolol in adult patients with elevated intraocular pressure: an eight-week, randomized, open-label, parallel-group, multicenter study in Latin America. Clin Ther. 2004;26(5): 755-68.

4. Malerbi FK, Hatanaka M, Vessani RM, Susanna R Jr. Intraocular pressure variability in patients who reached target intraocular pressure. Br J Ophthalmol. 2005;89(5):540-2.

5. Susanna R Jr, Vessani RM, Sakata L, Zacarias LC, Hatanaka M.The relation between intraocular pressure peak in the water drinking test and visual field progression in glaucoma. Br J Ophthalmol. 2005;89(10):1298-301.

6. Susanna R Jr, Hatanaka M, Vessani RM, Pinheiro A, Morita C. Correlation of asymmetric glaucomatous visual field damage and water-drinking test response. Invest Ophthalmol Vis Sci. 2006;47(2):641-4.

7. Vasconcelos-Moraes CG, Susanna R Jr. Correlation between the water drinking 
test and modified diurnal tension curve in untreated glaucomatous eyes. Clinics (Sao Paulo). 2008;63(4):433-6.

8. Hatanaka M, Grigera DE, Barbosa WL, Jordao M, Susanna R Jr. An eightweek, multicentric, randomized, interventional, open-label, phase 4, parallel comparison of the efficacy and tolerability of the fixed combination of timolol maleate $0.5 \%$ /brimonidine tartrate $0.2 \%$ versus fixed combination of timolol maleate $0.5 \%$ /dorzolamide $2 \%$ in patients with elevated intraocular pressure. $\mathrm{J}$ Glaucoma. 2008;17(8):674-9.

9. De Moraes CG, Reis AS, Cavalcante AF, Sano ME, Susanna R Jr. Choroidal expansion during the water drinking test. Graefes Arch Clin Exp Ophthalmol. 2009;247(3):385-9.

10. De Moraes CG, Furlanetto RL, Reis AS, Vegini F, Cavalcanti NF, Susanna R Jr. Agreement between stress intraocular pressure and long-term intraocular pressure measurements in primary open angle glaucoma. Clin Experiment Ophthalmol. 2009;37(3):270-4.

11. Hatanaka M, Reis A, Sano ME, Susanna R Jr. Additive intraocular pressure reduction effect of fixed combination of maleate timolol $0.5 \%$ /dorzolamide $2 \%$ (Cosopt) on monotherapy with latanoprost (Xalatan) in patients with elevated intraocular pressure: a prospective, 4-week, open-label, randomized, controlled clinical trial. J Glaucoma. 2009 Sep 2 [Epub ahead of print]

12. Kumar RS, de Guzman MH, Ong PY, Goldberg I. Does peak intraocular pressure measured by water drinking test reflect peak circadian levels? A pilot study. Clin Experiment Ophthalmol. 2008;36(4):312-5.

13. Mansouri K, Orguel S, Mermoud A, Haefliger I, Flammer J, Ravinet E, Shaarawy T. Quality of diurnal intraocular pressure control in primary open- angle patients treated with latanoprost compared with surgically treated glaucoma patients: a prospective trial. Br J Ophthalmol. 2008;92(3):332-6.

14. Danesh-Meyer HV. The water-drinking test: the elegance of simplicity. Clin Experiment Ophthalmol. 2008;36(4):301-3.

15. Danesh-Meyer HV, Papchenko T, Tan YW, Gamble GD Medically controlled glaucoma patients show greater increase in intraocular pressure than surgically controlled patients with the water drinking test. Ophthalmology. 2008;115(9):1566-70.

16. Guedes RA, Guedes VM, Chaoubah A. [Use of water drinking test after nonpenetrating deep sclerectomy.] J Fr Ophtalmol. 2005;28(10):1076-80. French

17. Vetrugno M, Sisto D, Trabucco T, Balducci F, Delle Noci N, Sborgia C. Water-drinking test in patients with primary open-angle glaucoma while treated with different topical medications. J Ocul Pharmacol Ther. 2005;21(3):250-7.

18. Christiansen GA, Nau CB, McLaren JW, Johnson DH. Mechanism of ocular hypotensive action of bimatoprost (Lumigan) in patients with ocular hypertension or glaucoma. Ophthalmology. 2004;111(9):1658-62.

19. Susanna R, Takahashi WY, Betinjane AJ. Reprodutibilidade da curva tensional diaria I. Arq Bras Oftalmol. 1979;42:275-6.

20. Takahashi WY, Susanna R, Betinjane AJ. Reprodutibilidade da curva tensional diaria II. Arq Bras Oftalmol. 1979;42:277-8.

21. Tosi M Silva, Moraes Silva MRB. Comparison of the values of peaks and fluctuations of pressure in 2 water druinking tests in different schedules. World Glaucoma Congress. Boston (MA);2009. Poster 285.

22. Yuttiham K. Estermann S, Chen J, Yang T, Lin S, Stamper RL. The relationship between progression of glaucoma and supine and water drinking tests. World Glaucoma Congress. Boston (MA);2009. Poster 282.

\section{Resposta}

\section{Replay}

\section{Caro Editor,}

Agradecemos o interesse do Dr. Vessani e colaboradores em nosso artigo "Reproducibility of the Water Drinking Test performed at Different Times of the Day". O objetivo desse estudo foi comparar o resultado do TSH realizado em diferentes horas do dia em pacientes com glaucoma e indivíduos normais. Como Dr. Vessani coloca, mesmo que o objetivo do teste seja avaliar a facilidade de escoamento do paciente e não realizar o diagnóstico de glaucoma, é importante que esse teste seja reprodutível independente da hora do dia em que seja realizado.

Os resultados de nosso estudo não revelam a reprodutibilidade esperada de um teste que se propõe a avaliar a facilidade de escoamento após uma sobrecarga hídrica. Apesar dos picos pressóricos apresentarem uma concordância razoável, com diferenças maiores que $3 \mathrm{mmHg}$ em $20 \%$ dos casos de pacientes glaucomatosos e $23 \%$ dos indivíduos normais, chama a atenção a grande proporção de pacientes que apresentou variações de pressão intraocular maiores que $3 \mathrm{mmHg}$ : $40 \%$ dos pacientes glaucomatosos e $13 \%$ dos indivíduos normais. Quando avaliamos diferenças maiores que $3 \mathrm{mmHg}$, vale dizer que isso equivale a detectar diferenças maiores ou iguais a $4 \mathrm{mmHg}$, uma variação altamente significativa. Se formos mais conservadores e avaliarmos diferenças maiores ou iguais a $2 \mathrm{mmHg}$, os números descritos anteriormente sobem $100 \%$ e $90 \%$ (diferença de pico para glaucomatosos e controles, respectivamente) e $80 \%$ e $70 \%$ (diferença de variação para glaucomatosos e controles, respectivamente).

A pergunta que se faz é prática: se um indivíduo glaucomatoso realiza um TSH e não mostra variação de PIO às 12 horas, devemos valorizá-lo? E se o mesmo paciente repete o teste às $17 \mathrm{~h}$ e tem uma variação de $7 \mathrm{mmHg}$ (como aconteceu no indivíduo ilustrado na Figura 3, gráfico C)? Qual dos testes deveremos valorizar? O que não apresentou variação, indicando um escoamento adequado, ou o que apresentou um aumento de $7 \mathrm{mmHg}$, sugerindo uma dificuldade de escoamento importante? São questões como essa que dificultam, em nossa análise, a aplicação do teste na rotina clínica.

Quanto aos vários estudos investigando a aplicabilidade do teste, isso destaca o interesse de vários autores em avaliar melhor essa ferramenta. Vale a pena dizer que nosso estudo não encerra o assunto e que novas investigações são muito bem-vindas no sentido de melhor identificar a área de aplicação do TSH.

Vital Paulino Costa Flávio MacCord Medina José Paulo C. Vasconcellos 